\section{GP254 MATERNAL AWARENESS, ACCEPTABILITY AND WILLINGNESS TOWARDS GROUP B STREPTOCOCCUS (GBS) VACCINATION DURING PREGNANCY}

${ }^{1}$ Siobhan McCormack*, 'Claire Thompson, ${ }^{1}$ Caitriona Ní Chathasaigh, ${ }^{2}$ Miriam Nolan, ${ }^{2}$ Mendinaro Imcha, ${ }^{3}$ Ann Dee, ${ }^{4}$ Jean Saunders, ${ }^{1,5}$ Roy K Philip. ${ }^{1}$ Division of Neonatology, Department of Paediatrics, University Maternity Hospital, Limerick, Ireland; ${ }^{2}$ Department of Obstetrics and Gynaecology, University Maternity Hospital, Limerick, Ireland; ${ }^{3}$ Department of Public Health, Health Service Executive, Limerick, Ireland; ${ }^{4}$ Statistical Consulting Unit, University of Limerick, Limerick, Ireland; ${ }^{5}$ Graduate Entry Medical School (GEMS), University of Limerick, Limerick, Ireland

\subsection{6/archdischild-2019-epa.313}

Background Asymptomatic carriage of GBS in Ireland affects 20-33\% of women. In 2017 seventy-five infants developed invasive GBS disease in Ireland, equating to over 1 in 1000 live births. Death from invasive GBS in infants ranges 2-10\% and long-term neurological complications occur in up to $50 \%$ of cases of GBS meningitis. GBS has been identified by the World Health Organisation (WHO) as a high priority for vaccine development and Vaccines for pregnant women to protect their new-born infants against GBS infection are currently being developed.

Aims To evaluate maternal knowledge of GBS infection and to assess likely acceptance if an approved licenced vaccine for use in pregnancy is made available in Ireland

Methods Pregnant women were surveyed prospectively during normal and high-risk antenatal clinic visits. Information leaflet was provided, consent for the survey obtained and ethical approval was granted by the UL Hospitals Group research ethics committee. Study design proposed 750 pregnant women for consecutive enrollment.

Results The responses from the initial part of the survey was analysed. Mean age of women surveyed was 32 years (range 17-42 years) while gestation ranged from 11 to 40 weeks of gestation (mean 29 weeks). 57\% of women were in their first pregnancy, $20 \%$ in their second and the remaining $23 \%$ in their third or subsequent pregnancies. $88 \%$ had GBS status ascertained prior to study with $26 \%$ positive, and $74 \%$ tested clear for GBS.

$35 \%$ of women had never heard of GBS, $29 \%$ had some knowledge of it $29 \%$ had some knowledge of it including its significance in newborn babies and $7 \%$ had previous experience of it personally.

$35 \%$ of women felt that recommended vaccines would protect their baby from illness, $21 \%$ felt confident in recommended vaccines, and a further $29 \%$ both felt that vaccines would protect their baby from illness, and they felt confident in recommended vaccines. A combined positive response from $85 \%$ of pregnant women.

$54 \%$ indicated a discussion with their GP best influencing their decision making, $23 \%$ preferred midwife, $14 \%$ opted for discussion with obstetrician, 4\% information leaflets, 3\% depended on a family member and $1 \%$ online resources.

Conclusion Over two thirds of pregnant women indicated willingness to accept a vaccine to protect their infants with high rates of vaccine confidence. Women's preferences for advice regarding vaccination during pregnancy should be considered when planning such programmes given the strong desire for information seeking from primary care setting.

\section{GP255 'MYCOOMBE' MHEALTH APP - A PILOT STUDY}

${ }^{1}$ Robert McGrath*, ${ }^{2}$ Tina Barrett, ${ }^{2}$ Liam O'Cuiv, 'Georsan Caruth, ${ }^{2}$ Aisling Walsh, ${ }^{2}$ Regien Biesma, ${ }^{3}$ Michael Turner, 'Jan Miletin, ${ }^{1}$ Anne Doolan. ${ }^{1}$ Department of Neonatology, Coombe Women and Infants University Hospital, Dublin, Ireland; ${ }^{2}$ Department of Epidemiology and Public Health Medicine, Royal College of Surgeons in Ireland, Dublin, Ireland; ${ }^{3}$ Department of Obstetrics and Gynaecology, Coombe Women and Infants University Hospital, Dublin, Ireland

\subsection{6/archdischild-2019-epa.314}

Background Over the past decade, mobile health (mHealth) has become a burgeoning field within public health. mHealth applications (apps) are software that are incorporated into smartphones to improve health outcome and health care services. A number of mHealth apps are focused on maternal and child health. The quality of and evidence base for the information these provide can be unreliable and not in line local healthcare practices.

Our aim was to perform a pilot study to determine the feasibility of using a unique mHealth app 'MyCoombe' in a cohort of new mothers and assess the acceptability of this app to study participants.

Methods We developed a unique mHealth application 'MyCoombe' for Android, the content of which was focused on newborn health and early development, newborn feeding and maternal postpartum health and drawn from reliable evidence based sources, which were in line with local healthcare practices.

Ethical approval was obtained from the Coombe Women \& Infants University Hospital (CWIUH) Research Ethics Committee. We then performed a prospective observational cohort study within CWIUH from April to November 2017.

Mothers attending routine antenatal clinics were approached, provided with study information and written informed consent obtained. Patient demographics were recorded and a link to download 'MyCoombe' was provided to study participants. At six weeks postpartum, a feedback survey was sent to all active participants.

Results We recruited 116 mothers in the study period, each of whom were given a download link to 'MyCoombe'. Forty percent (46/116) downloaded the app and logged in at least once. The app was accessed 289 times in total, for an average of 6.28 interactions per participant. These 46 were sent a link to a feedback survey. Twenty-six percent (12/46) participants completed the survey. Fifty percent of respondents reported that the app was missing features they required. One respondent reported that it had too much information, while one respondent stated that the app should have more information and topics to be more acceptable to the user. Sixty-six percent of respondents stated that they would recommend 'MyCoombe' to friends and family.

Conclusion The information needs of new mothers are multifaceted and vary significantly between individuals. While it was feasible to use 'MyCoombe' in a cohort of new mothers, there was significant participant attrition after recruitment with $60 \%(70 / 116)$ not downloading the app. Of survey responders $66 \%$ percent $(8 / 12)$ found the app to be acceptable and would recommend to friends and family. 